\title{
AN EXPERIMENTAL EVALUATION OF INTELLIGENT FAULT DETECTION AND CLASSIFICATION FOR INDUCTION MOTORS UTILIZING MACHINE LEARNING APPROACHES
}

\author{
Mahmut KASAP $^{1 *}$, Eyüp ÇİNAR ${ }^{2}$, Ahmet YAZICI $^{3}$, Kemal ÖZKAN ${ }^{4}$ \\ ${ }^{1}$ Gazi University, TUSAŞ-Kazan Vocational School, Computer Technologies, Ankara, \\ ORCID No: http://orcid.org/0000-0002-7213-1363
}

${ }^{2}$ Eskişehir Osmangazi University, Engineering and Architecture Faculty, Computer Engineering Department, Eskişehir, ORCID No : http://orcid.org/0000-0003-3189-7247

${ }^{3}$ Eskişehir Osmangazi University, Engineering and Architecture Faculty, Computer Engineering Department, Eskişehir, ORCID No: http://orcid.org/0000-0001-5589-2032

${ }^{4}$ Eskişehir Osmangazi University, Engineering and Architecture Faculty, Computer Engineering Department, Eskişehir, ORCID No: http://orcid.org/0000-0003-2252-2128

\begin{tabular}{ll}
\hline Keywords & Abstract \\
\hline Predictive maintenance & Maintenance planning is critical for efficient operations of manufacturing systems. While \\
Machine learning & unnecessary maintenance causes waste of money and time, skipping necessary \\
Vibration analysis & maintenance can also cause unexpected down times in production. Predictive \\
& maintenance activities which focus on both detection and classification of equipment \\
& faults at an early stage are classified under Condition-Based Maintenance. On the other \\
& hand, forecasting remaining useful life of equipment is classified under Prognostics. In \\
our study, fault detection and diagnosis of induction motors which are widely used in & factories for different purposes are targeted. Triaxial vibration data collected from two \\
& similar induction motors under different operating conditions are examined for potential \\
& failure scenarios. Various features of vibration data are extracted, scaled and labeled \\
& with operational status information. The obtained dataset is analyzed with six different \\
machine learning algorithms. Model performances are examined and compared against \\
each other. Our experimental results show that the abnormal operating conditions of \\
induction motors can be successfully detected utilizing machine learning algorithms.
\end{tabular}

\section{MAKİNE ÖĞRENMESI YAKLAŞIMLARI İLE İNDÜKSIYON MOTORLARI İÇIN AKILLI} HATA TESPITI VE SINIFLANDIRMADA DENEYSEL BIR DEĞERLENDİME

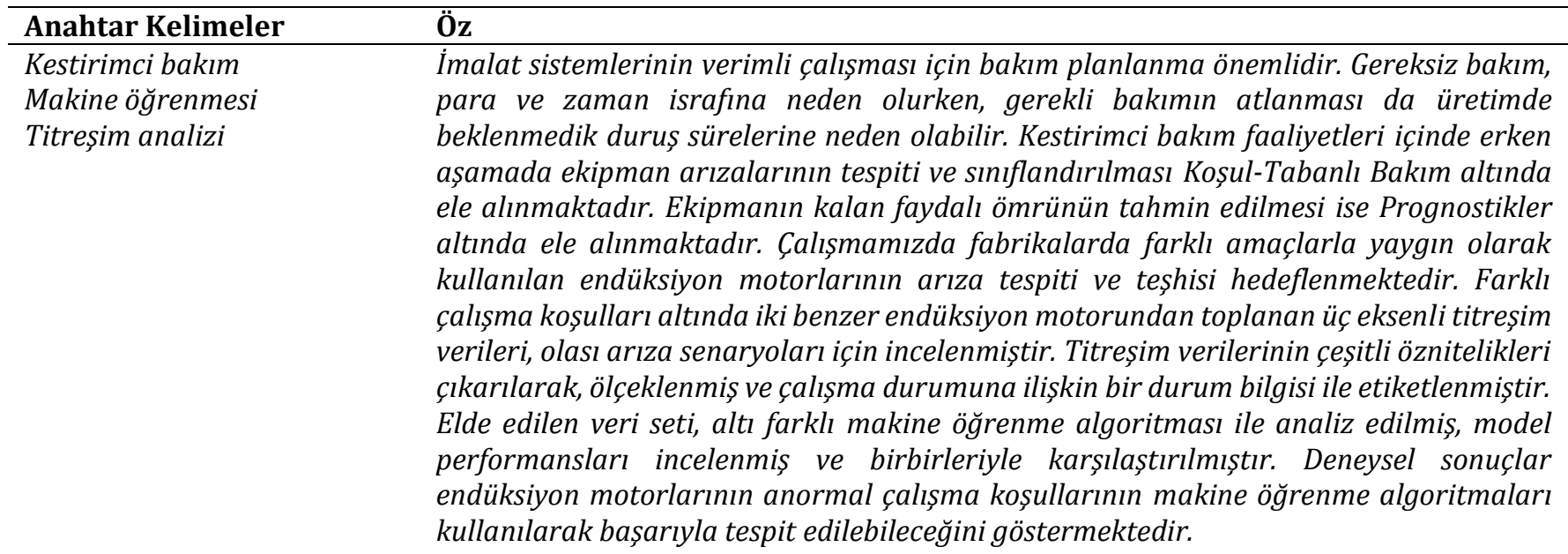

* Sorumlu yazar; e-posta : mahmutkasap@gazi.edu.tr

$\mathrm{Bu}$ eser, Creative Commons Attribution License (http://creativecommons.org/licenses/by/4.0/) hükümlerine göre açık erişimli bir makaledir.

This is an open access article under the terms of the Creative Commons Attribution License (http://creativecommons.org/licenses/by/4.0/). 


\begin{tabular}{llll}
\hline Araştırma Makalesi & & Research Article & \\
Başvuru Tarihi & $: 03.01 .2021$ & Submission Date & $: 03.01 .2021$ \\
Kabul Tarihi & $: 29.04 .2021$ & Accepted Date & $: 29.04 .2021$ \\
\hline
\end{tabular}

\section{Introduction}

Many types of equipment are used in production today. The uptime of these and their health state significantly affects manufacturing capacity and quality. To maximize equipment uptime, a comprehensive maintenance program is crucial for the factories.

Different maintenance strategies have been proposed in the literature. The general maintenance strategies are summarized in Figure 1 (Jimenez, Schwartz, Vingerhoeds, Grabot, and Salaün, 2020). Reactive (or after failure) approach refers to a maintenance when equipment breakdown occurs. For scheduled (preventive) maintenance, the main goal is to perform the maintenance on a schedule-basis to prevent an actual equipment failure occurrence. Preventive maintenance can be done periodically but frequent maintenance of a healthy equipment is generally undesirable due to cost. Predictive maintenance (PdM) suggests planning optimal maintenance intervals, taking into account the time and cost appropriate to the current state of the device. It includes both conditionbased maintenance and prognostics and health management. The former term focus on fault detection/diagnosis of equipment, while the later terms focused on forecasting remaining useful life of the equipment. The later stage of PdM activities are also generally referred as Prognostics (Carvalho et al., 2019).

A predictive maintenance program can reduce maintenance costs by $25 \%-35 \%$, eliminate breakdowns by $70 \%-75 \%$, reduce downtime by $35 \%-45 \%$ and increase production capacity by $25 \%-35 \%$ (Sullivan, Pugh, Melendez, and Hunt, 2010). Therefore, PdM technologies are getting increasingly popular among manufacturers today.

Faults might show varying symptoms on an equipment in different periods. At an early stage, lower noise and smaller amplitude of vibrations at different frequencies may be evident, as the severity of fault level increases these symptoms might become more dominant. Detecting these fingerprints at an early stage and projecting a lifetime for the faulty part is often a challenging task by maintenance operators. In recent years, it has been shown that data-driven Machine learning (ML) algorithms can achieve significant results at these tasks. One way to employ an ML algorithm for this task is to collect data representing both healthy baseline state and anomalous behavior and let the ML algorithm discriminate between faulty equipment states.

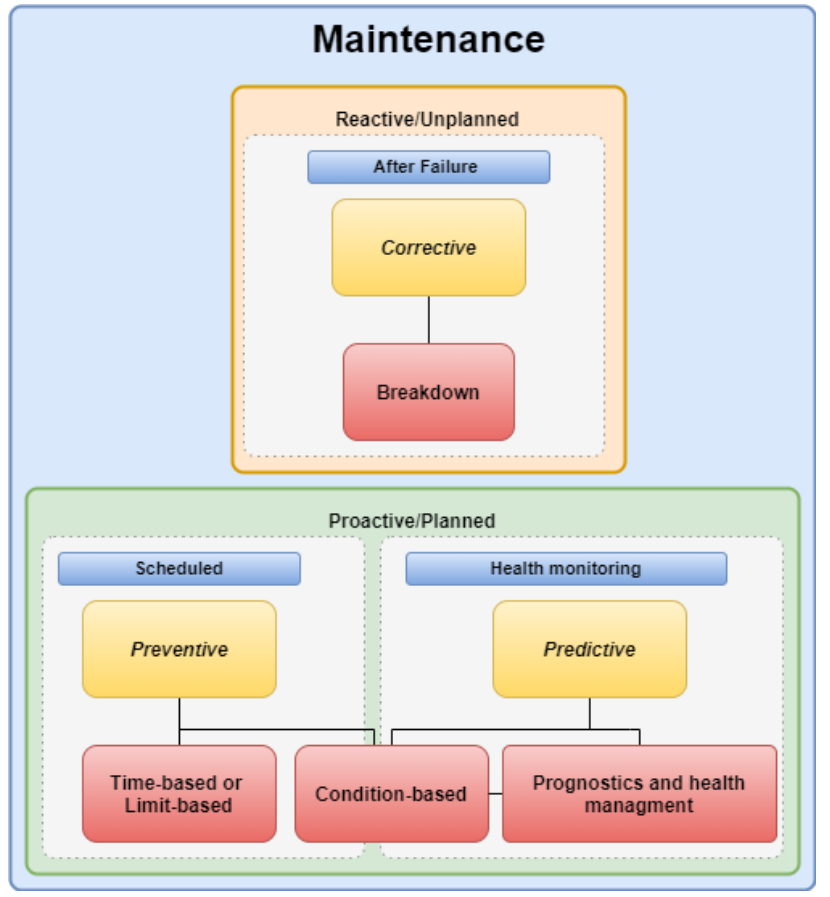

Figure 1. Maintenance Approaches

Various types of induction motors are widely used in manufacturing and therefore failure analysis of induction motors might be important (Benbouzid, 2000). Some of the common fault causes seen in induction motors are listed as follows (Kumar and Hati, 2021):

- Overloading

- Overspeed

- Manufacturing defects

- Dirt, debris and corrosion

- Component failures

- Overheating in winding and bearing

- Excessive dielectric stress

- Shock loads

In order to detect these faults studies generally focus on vibration (Rodriguez, Belahcen, and Arkkio, 2006). Velocity, acceleration or displacement due to mechanical faults reflects into vibration signal (Katalin, 2015).

Our motivation in this study is to successfully define abnormal operating conditions of induction motors used in air fans by experimentally examining their historical vibration data via multiple ML algorithms. By collecting time-series data at different stages of operating conditions, we demonstrate successful 
classification of these anomalous stages which provides a promising use-case scenario for a PdM application.

\section{Literature Review}

There are various types of ML models that can be employed in a PdM application. Supervised learning describes the process of learning the relationship between training data (input) and labeled data (output). On the other hand, unsupervised learning methods do not require labeling and operates based on spatial distribution of data set.

ML based fault diagnosis has been studied frequently in the literature in recent years. Deng et al. have proposed empirical mode decomposition, fuzzy information entropy, improved particle swarm optimization (PSO) algorithm and least squares support vector machines (LS-SVM) for motor bearing fault diagnosis (Deng, Yao, Zhao, Yang, and Li, 2019). Liu et al. have proposed a hybrid intelligent method for multi-fault detection of rotating machines using redundant second-generation wavelet packet transformation (RSGWPT), kernel principal component analysis (KPCA) and twin support vector machine (TWSVM) and found the method effective in their experiments (Liu, Guo, $\mathrm{Hu}$, and $\mathrm{Ma}$, 2017). Support vector machine (SVM) based on the modified shuffled frog-leaping algorithm (MSFLA) has been designed for an efficient fault classification (You et al., 2019). In another study, Wang proposed an advanced knn-based method to detect different gear cracks at different loads and speeds (Wang, 2016). Glowacz proposed a method of extracting attributes from acoustic signals then using the method for singlephase induction motor fault diagnosis with the nearest neighbor (Glowacz, 2019). Zhou et al. have proposed an isolation index provided by decomposing the $\mathrm{kNN}$ distance used as the detection index in $\mathrm{kNN}$-based fault detection method (Zhou, Wen, and Yang, 2016). In another work, Saravanan and Ramachandran investigated the use of discrete wavelets for feature extraction and a Decision Tree for classification (Saravanan and Ramachandran, 2009).

In our study, we aim to classify the working conditions of induction motors with a real-world experimental data collected in a lab environment by utilizing various supervised learning algorithms and comparing them against each other. The Supervised ML algorithms explored in this study include SVM, Logistic and Ridge Regressions, KNN and Ensemble Methods including Bagging and Random Forests.

Supervised ML algorithms are among the most widely used data-driven methods in PdM. However other types of ML algorithms under the field of Artificial Intelligence might be also employed. Unsupervised algorithms can be preferred in cases where dataset labelling is not required (Alpaydin, 2020). An ontological figure representing the set of algorithms under ML field is shown in Fig. 2.

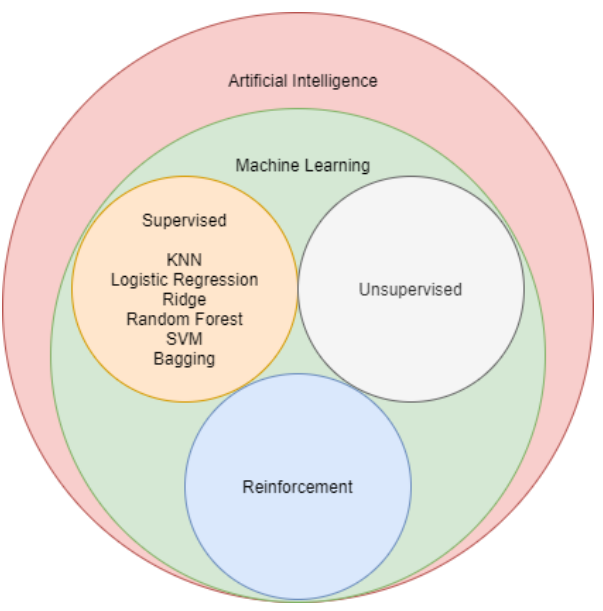

Figure 2. Algorithms Used in ML

Among the popular supervised learning algorithms, Support Vector Machine (SVM) can be defined as a vector space-based ML method that finds a decision boundary between two or more classes in training data. Support vector machines are generally used to separate data that consist of two classes, for example, to separate each data in a data set as patient or healthy. For multiclass problem cases, it is possible to train different SVMs for each class or for each class pair. SVM tries to create the optimal hyperplane that classifies the data set as shown in Figure 3. A comparison study on SVM and popular classification algorithms for intelligent diagnosis of rotating machines is given in (Han, Jiang, Zhao, Wang, and Yin, 2017). In another study, the authors used SVM for acceleration data and rub malfunctions in their studies (Fengqi and Meng, 2006).

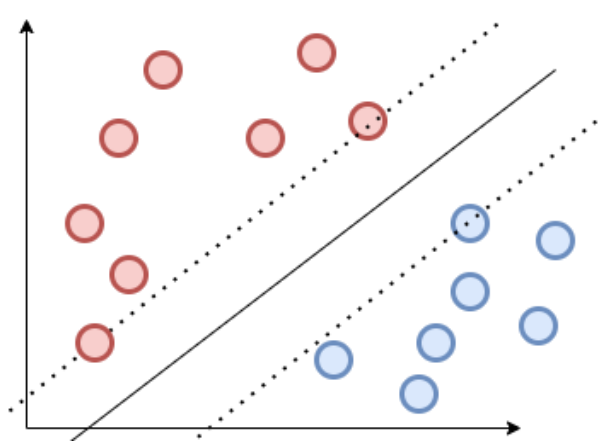

Figure 3. SVM Hyperplane

Logistic Regression is a regression method that can be used for classification tasks. It is used to classify categorical or numerical data via a logistic function curve as shown in Figure 4. Logistic regression is 
generally used to separate binary class data but in a multi-class problem, each class can be evaluated as a binary classification according to all other classes (one vs all method).

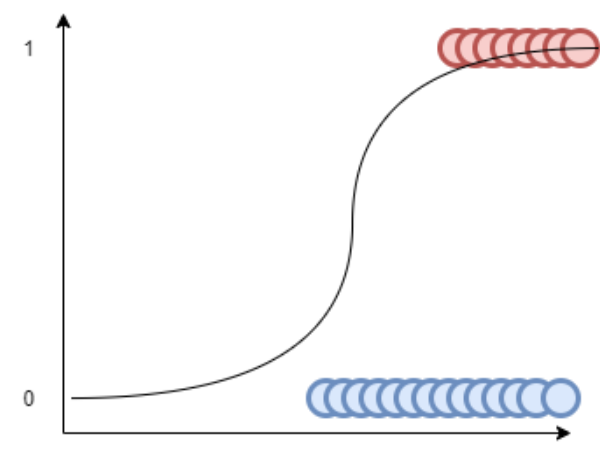

Figure 4. Logistic Regression Logistic Function Curve

Saunders et al. made a brief description in their work for ridge regression (Saunders, Gammerman, and Vovk, 1998). A training set $\left(x_{1}, y_{1}\right), \ldots,\left(x_{M}, y_{M}\right)$ where $M$ is the number of examples $x_{M}$ are vectors in $\mathbb{R}^{n}$ and $y_{m} \in$ $\mathbb{R}^{n} m=1, \ldots, M$ comparison class consists of the linear functions $y=w \cdot x$ where $w \in \mathbb{R}^{n}$ the Least Squares method recommends computing $w=w_{0}$ which minimizes

$$
L_{M}(w)=\sum_{m=1}^{M}\left(y_{m}-w \cdot x_{m}\right)^{2}
$$

and using $\mathrm{w}_{0}$ for labeling future examples. For example, if a new example has attributes $\mathrm{x}$, the predicted label is $w_{0} \cdot x$.

The Ridge Regression replaces the objective function $L_{M}(w)$ with

$$
\alpha\|w\|^{2}+\sum_{m=1}^{M}\left(y_{m}-w \cdot x_{m}\right)^{2}
$$

where $\alpha$ is a fixed positive constant.

On the other hand, Ridge regression-based classification aims to solve the objective in (2) as a regression task in the form of binary classification.

In ensemble algorithms, Bagging methods utilizes a set of base models that create several samples of an estimator on random subsets of the training set, and then combine their individual predictions to generate a final prediction as shown in Fig. 5. The base model used with Bagging in our study is Decision Trees.

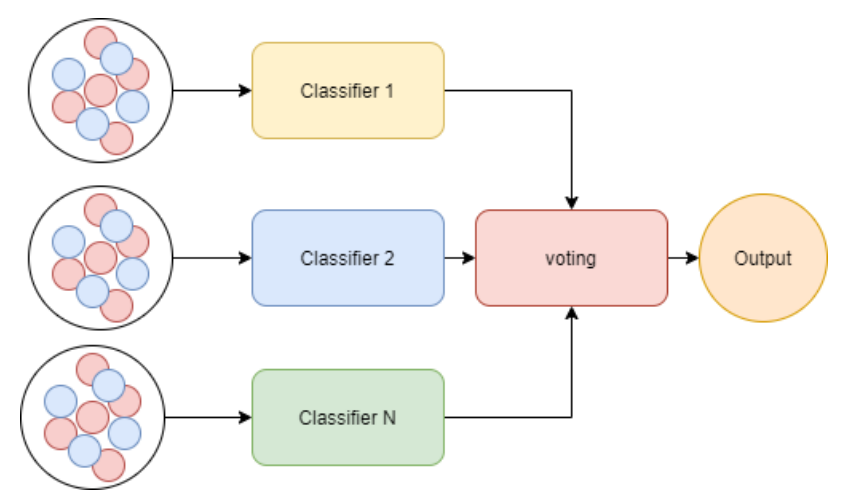

Figure 5. Bagging Architecture

Random Forest is one of the popular ML models more generally known as Ensemble methods (Figure 6). Its robustness to overfitting and noise usually gives superior performance in ML tasks. Random forest selects and trains many random different decision trees. By this way, many decision trees are created, and each decision tree makes individual predictions. At the end of these processes, if the problem is a regression problem, average of the decision tree estimates are taken, if the problem is a classification problem then a voting mechanism is employed to select the most popular class. When separating each node during the construction of a tree, the best separation is found from all input properties or a random size subset. The purpose of this randomness process is to reduce the variance of the Random Forest classifier. A fault diagnosis study has been carried out with PCA and decision tree, authors stated that their methods have higher accuracy and less training time than back propagation neural network (BPNN) (Sun, Chen, and Li, 2007).

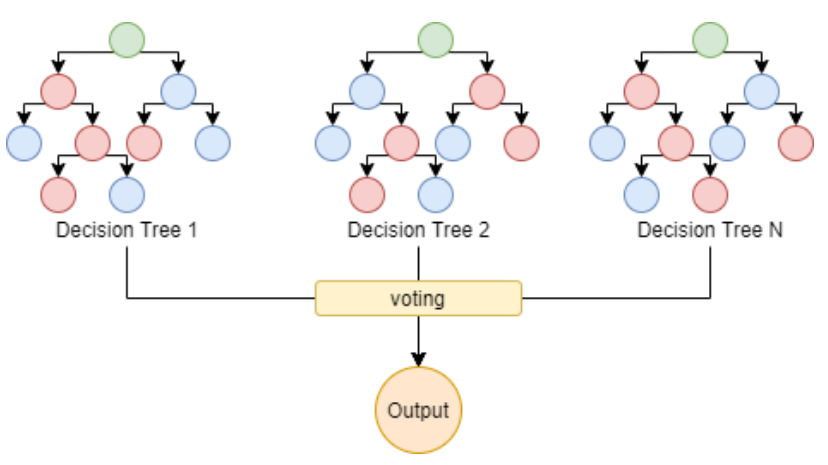

Figure 6. Random Forest Architecture

K-Nearest Neighbors classification is a type of examplebased learning method. Classification calculates the nearest neighbors of each point by simple majority vote 
and assigns them to the class with the most representatives. A representative figure is given in Fig. 7. Various distance metrics can be used for k-NN such as Euclidean, Manhattan. Moosavian et al. proposed an approach for detecting unbalanced fault in rotating machines using KNN and SVM classifiers (Moosavian, Ahmadi, Sakhaei, and Labbafi, 2014).

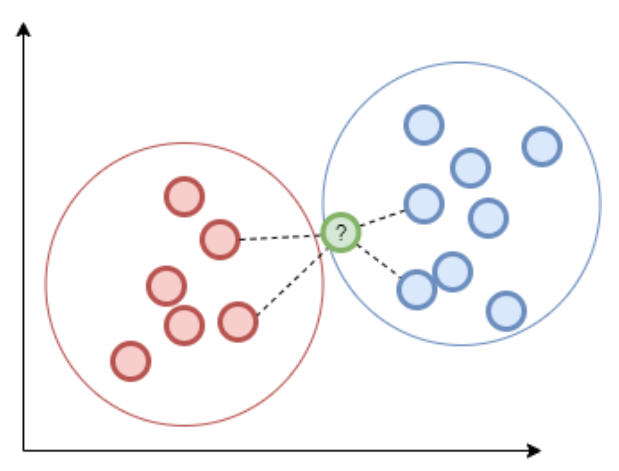

Figure 7. KNN Distance

\section{Method}

The study progressed in the stages of preparation, feature extraction, scaling, train and test split, training, evaluation and tuning with train data, testing with offline test data. These steps are shown in Figure 8.

The authors declared that research and publication ethics were followed in this study.

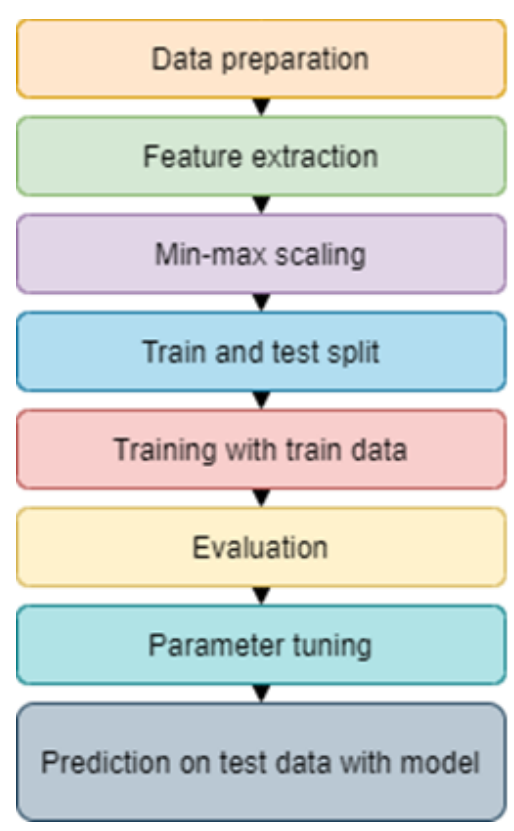

Figure 8. General Approach Followed in The Study

\subsection{Data Collection and Test System}

An experimental data is collected for Predictive Maintenance studies by Lider Teknoloji Geliştirme company. It also permits to use the data for publication. Data is collected simultaneously from two identical induction fan motors with National Instruments (DAQ) system and vibration sensors ADXL326(+-16g). Test setup picture and representative visual diagram can be seen in Figure 9.

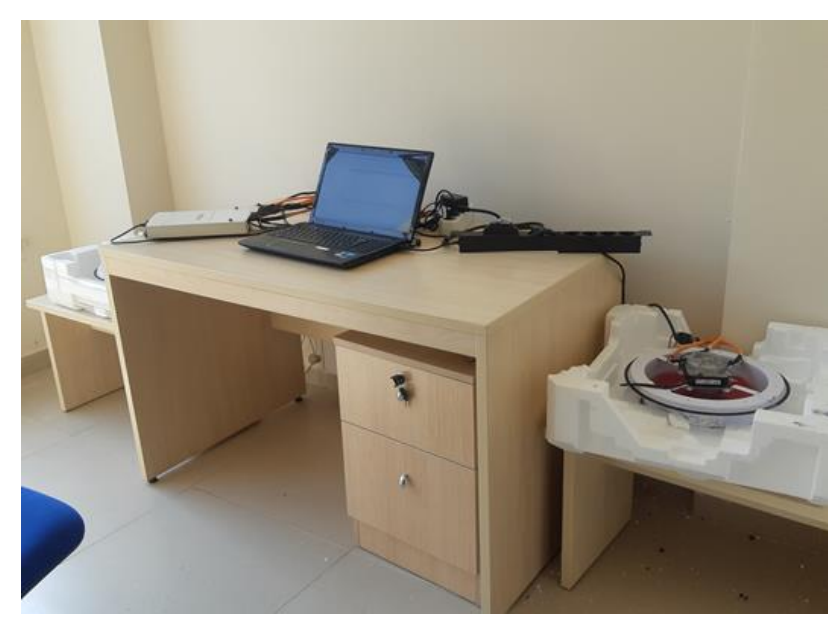

(a)

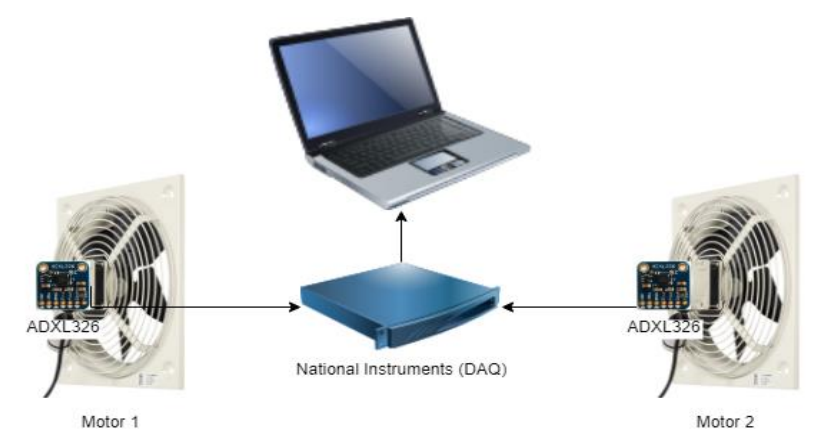

(b)

Figure 9. (a) Test Setup Picture For Two Identical Induction Motors; (B) Test Setup Representative Visual Diagram.

Data set contains a total of 840 seconds three axis vibration measurement signal. The vibration data for each axis is sampled at $10 \mathrm{kHz}$ and tagged with timestamps over recording time. While one of fan motors is continuously operated under normal conditions, the other fan was driven in 9 different overloaded operating conditions with different time periods (Table 3). Nine different class of operating conditions are created via modifications that cause the fan blades in the engine propeller to rub against a styrofoam housing in which the fan is placed. The friction amount is increased by modifications to the fan blades. 


\subsection{Experiments}

The three axis vibration data in the data set is divided into $1 \mathrm{kHz}$ sample windows. The features given in Table 1 are extracted for each window. Then, the extracted features are scaled between 0-1 using the min-max method. The extracted vibration features are labeled with a class label corresponding to specific working condition. Whole dataset is divided as 70\% training and $30 \%$ test data with stratified sampling to minimize class imbalance.

The classification problem is considered two ways. First, as a binary classification problem regardless of operation condition which includes discriminating normal (healthy) operation against any abnormal (anomaly) operation. Second, as a multi-class approach discriminating against each artificially generated class (total of 10 classes). According to the first approach, labeling and class distribution are shown in Table 2 .

\section{Table 1}

Extracted Features From Raw Vibration Data

\begin{aligned} & \hline No Feature \\ & \hline 1 Root Mean Square \\ & 2 Max Absolute Amplitude \\ & 3 Skewness \\ & 4 Kurtosis \\ & 5 Crest Factor \\ & 6 Impact Factor \\ & 7 Max Amplitude \\ & 8 Min Amplitude \\ & 9 Peak to Peak Amplitude \\ & 10 Mean \\ & 11 Median \\ & 12 Mean Absolute \\ & 13 Amplitude \\ & 14 Wariance \\ & 15 Peak Index \\ & 16 Impluse Factor \\ & \hline\end{aligned}

A sample raw vibration data for $\mathrm{Y}$ axis is given in Figure 10.a. The unscaled values of features are also shown in Figure 10.b. Feature values on other axes are also in similar ranges.

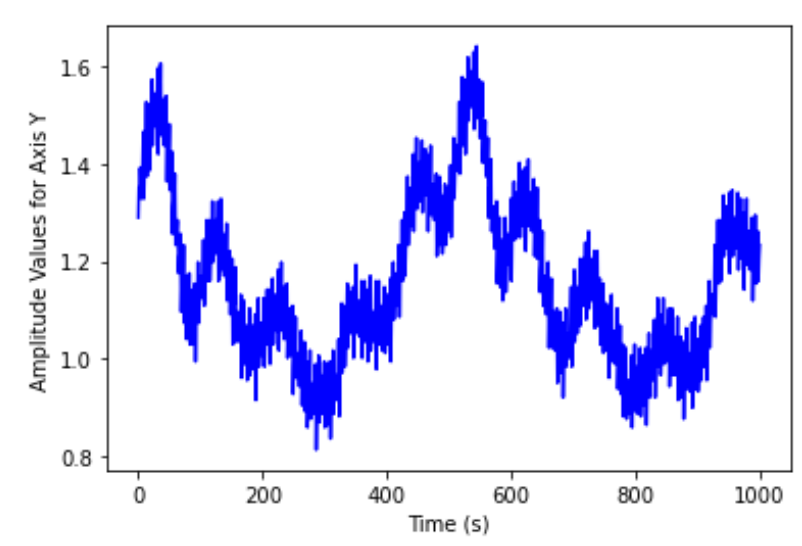

(a)

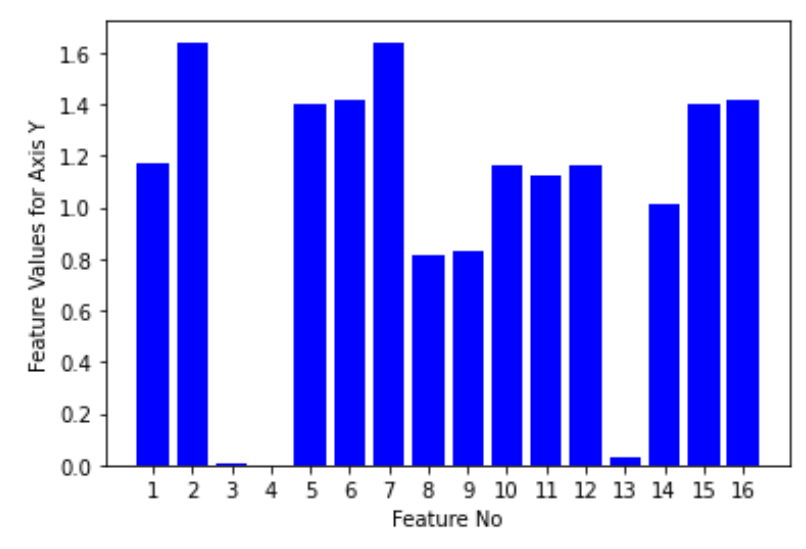

(b)

Figure 10. (a) Sample Raw Vibration Data Window For Y Axis; (B) Sample Feature Values (before scale progress)

Table 2

Working Conditions, Class Numbers And The Number Of Windows In The Training And Test Data For BinaryClass Approach

\begin{tabular}{|c|c|c|c|c|}
\hline Class & Condition & Motor & $\begin{array}{l}\text { Training } \\
\text { Window }\end{array}$ & $\begin{array}{c}\text { Test } \\
\text { Window }\end{array}$ \\
\hline 1 & $\begin{array}{c}\text { Under } \\
\text { normal } \\
\text { condition }\end{array}$ & $\begin{array}{c}1 \text { and } \\
2\end{array}$ & 2945 & 1275 \\
\hline \multirow[t]{2}{*}{2} & $\begin{array}{l}\text { Abnormal } \\
\text { operating } \\
\text { condition }\end{array}$ & 2 & 2935 & 1245 \\
\hline & & Total & 5880 & 2520 \\
\hline
\end{tabular}

In the second approach, the details of abnormal operating conditions are also used as label information as shown in Table 3 . Total of 10 different classes (including motors normal condition classes) and their class distribution is also presented in Table 3.

In training of each algorithm, 70\% of the training dataset are used. Test classification scores are calculated using 
the model that achieves the highest training accuracy score.

\section{Table 3}

Working Conditions, Class Numbers And The Number Of Windows In The Training And Test Data For Multi-Class Approach

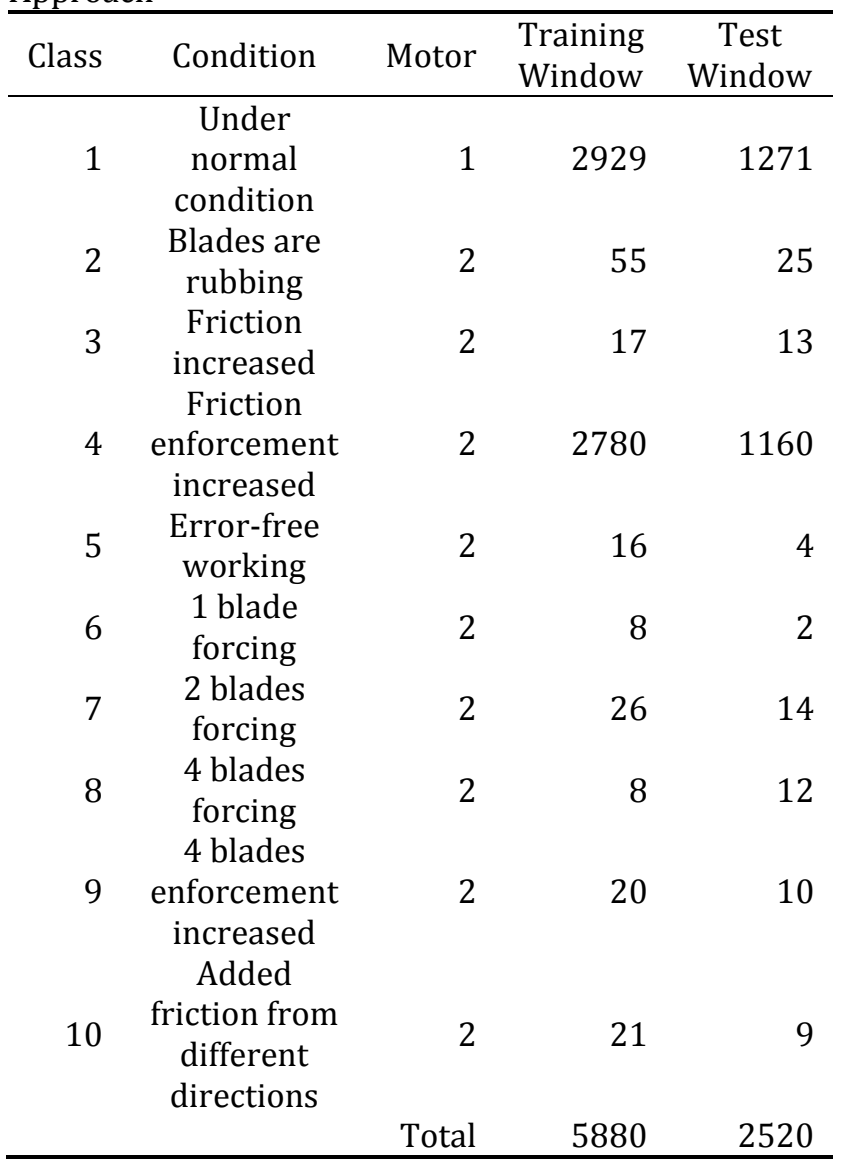

\section{Results}

The test results for the binary-class approach are listed below (Table 4, Figure 11 and Figure 13). We observe that SVM and Logistic Regression algorithms are slightly better with test data.

The test results for the multi-class approach are listed below (Table 5 and Figure 12). Looking at the results, we see that although the precision macro avg score for Logistic Regression (0.970256) is high, the precision weighted avg score is 0.998714 and is slightly lower than the score of Random Forest and SVM (0.998820). Likewise, the recall macro avg, f1 macro avg and f1 weighted avg scores are also slightly lower than the SVM and Random Forest score. Considering that our focus in this study is also the detection of particular anomalous behavior types, we find Random Forest and SVM more successful as can be seen from the confusion matrix in Figure 14.

The results obtained from the experiments show that operating conditions of induction fan motors can be classified highly successfully via ML algorithms. Formulating the problem as a binary-case problem would be sufficient to determine whether the equipment is running under normal or abnormal condition. However, if particular operating condition is point of interest then in that case, we also showed that formulating the problem as a multi-class can also achieve significantly high success rates. In the binaryclass approach, it can be said that most algorithms give good results. In the multi-class approach, SVM and Random Forest showed slightly more successful test results in classifying abnormal situations.

Table 4

Algorithms Test Scores For Binary-Class

\begin{tabular}{lcccccc}
\hline & $\begin{array}{c}\text { Logistic } \\
\text { Algorithm }\end{array}$ & $\begin{array}{c}\text { Ridge } \\
\text { Regression }\end{array}$ & Classifier & K-NN & Bagging & \multicolumn{2}{c}{ Random } \\
Forest & SVM \\
\hline Accuracy & $\mathbf{0 . 9 9 9 6 0 3}$ & 0.998413 & 0.999206 & 0.999206 & 0.999206 & $\mathbf{0 . 9 9 9 6 0 3}$ \\
Cohen Kappa & $\mathbf{0 . 9 9 9 2 0 6}$ & 0.996825 & 0.998412 & 0.998413 & 0.998413 & $\mathbf{0 . 9 9 9 2 0 6}$ \\
Precision macro avg & $\mathbf{0 . 9 9 9 6 0 8}$ & 0.998399 & 0.999206 & 0.999198 & 0.999198 & $\mathbf{0 . 9 9 9 6 0 8}$ \\
Precision weighted avg & $\mathbf{0 . 9 9 9 6 0 3}$ & 0.998418 & 0.999206 & 0.999208 & 0.999208 & $\mathbf{0 . 9 9 9 6 0 3}$ \\
Recall macro avg & $\mathbf{0 . 9 9 9 5 9 8}$ & 0.998431 & 0.999206 & 0.999216 & 0.999216 & $\mathbf{0 . 9 9 9 5 9 8}$ \\
Recall weighted avg & $\mathbf{0 . 9 9 9 6 0 3}$ & 0.998413 & 0.999206 & 0.999206 & 0.999206 & $\mathbf{0 . 9 9 9 6 0 3}$ \\
f1 macro avg & $\mathbf{0 . 9 9 9 6 0 3}$ & 0.998413 & 0.999206 & 0.999206 & 0.999206 & $\mathbf{0 . 9 9 9 6 0 3}$ \\
f1 weighted avg & $\mathbf{0 . 9 9 9 6 0 3}$ & 0.998413 & 0.999206 & 0.999206 & 0.999206 & $\mathbf{0 . 9 9 9 6 0 3}$ \\
\hline
\end{tabular}




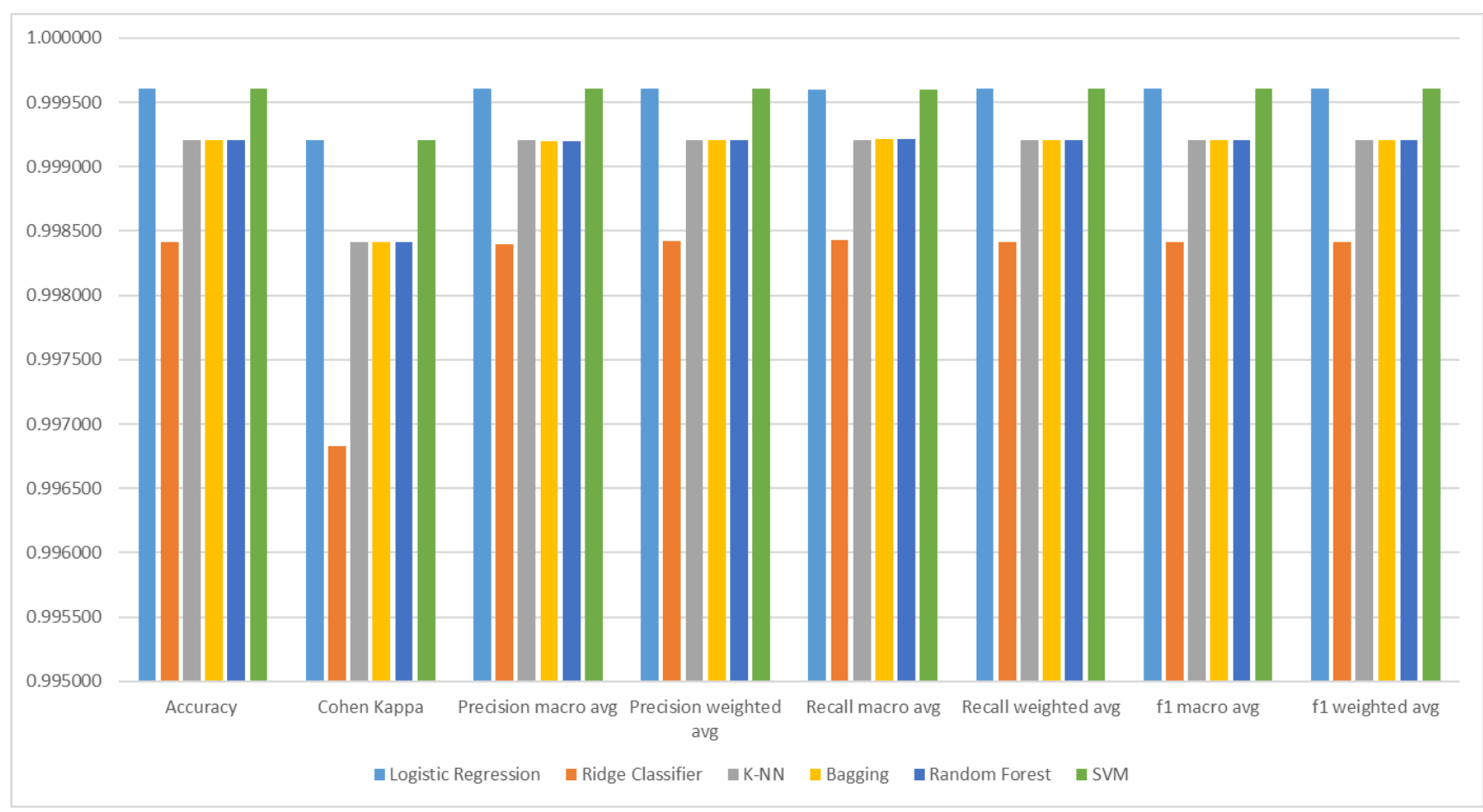

Figure 11. Algorithms Training Scores For Binary-Class

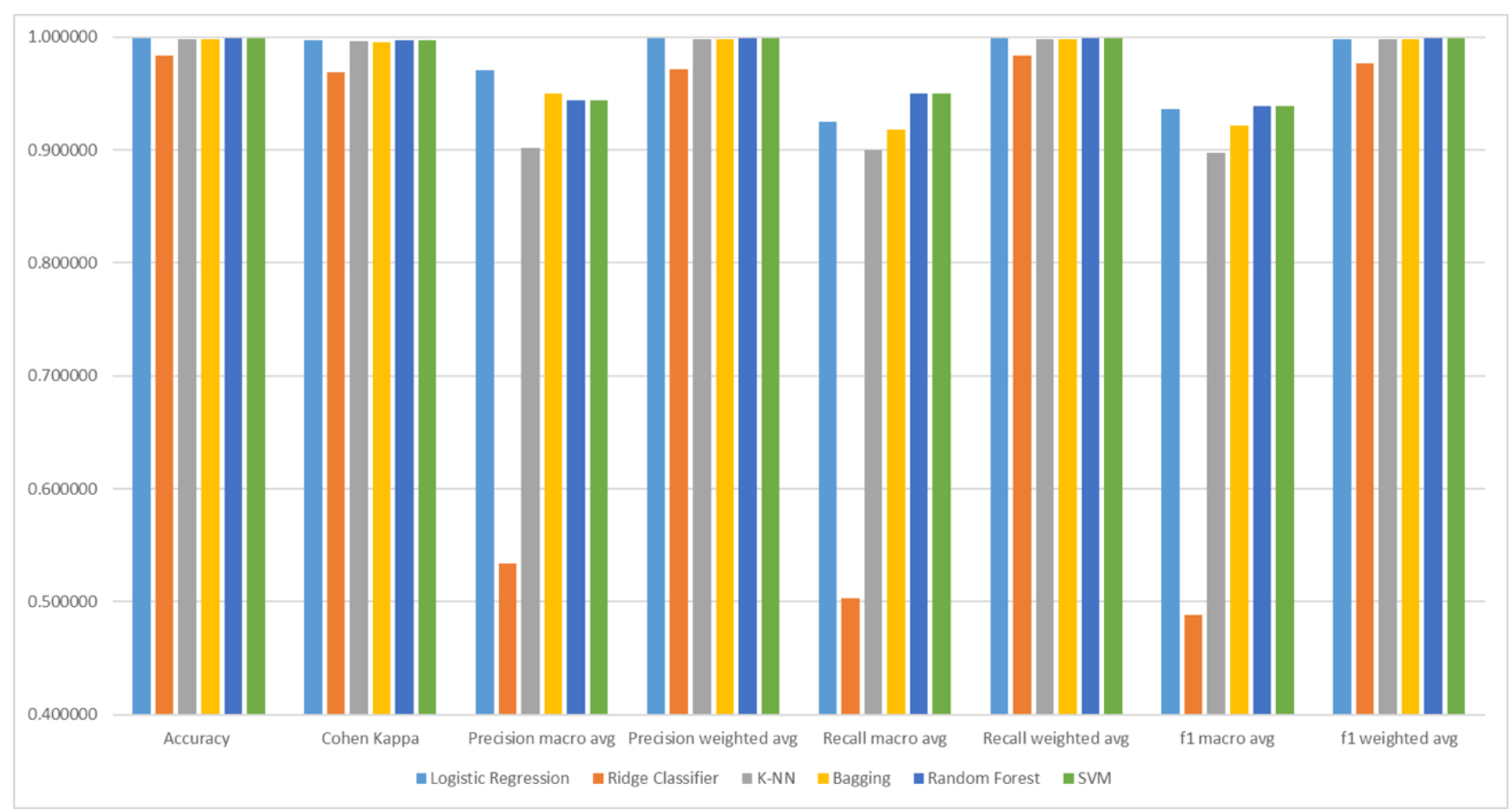

Figure 12. Algorithms Test Scores For Multi-Class 
Figure 13 shows the confusion matrix of algorithms for the binary-class approach, while Figure 14 shows the results of the multi-class approach.

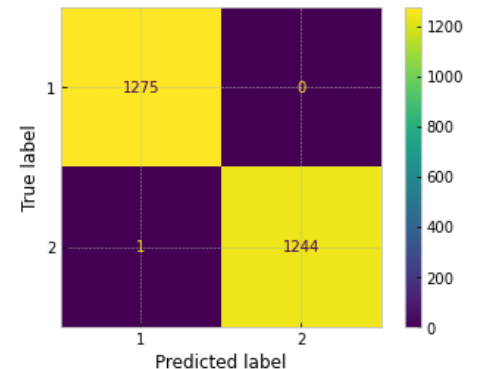

(a)

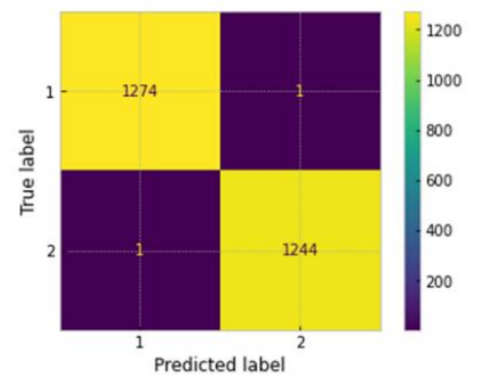

(c)

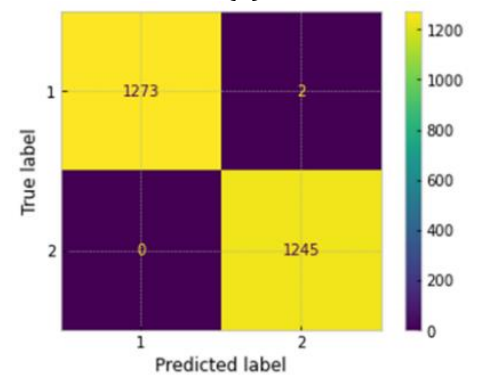

(e)

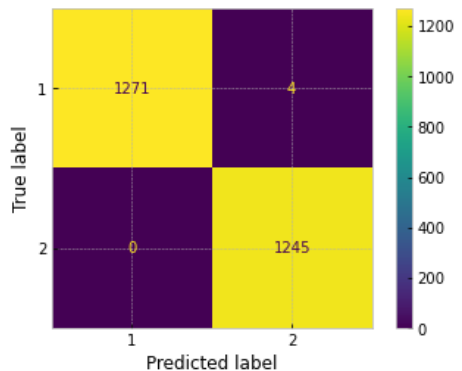

(b)

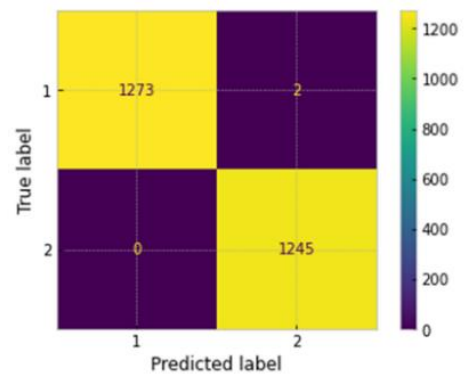

(d)

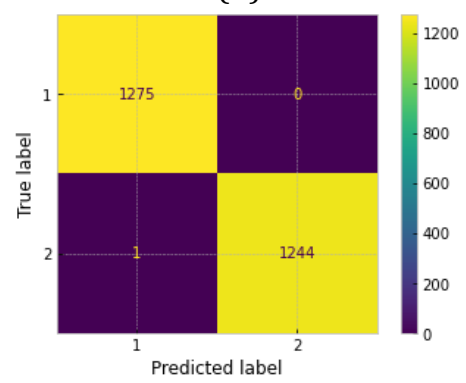

(f)

Figure 13. Confusion Matrix For Binary-Class Tests: (A) Logistic Regression; (B) Ridge Classifier; (C) K-NN ; (D) Bagging; (E) Random Forest; (f) SVM;

Table 5

Algorithms Test Scores For Multi-Class

\begin{tabular}{lrcrrrr}
\hline Algorithm & $\begin{array}{c}\text { Logistic } \\
\text { Regression }\end{array}$ & $\begin{array}{c}\text { Ridge } \\
\text { Classifier }\end{array}$ & K-NN & \multicolumn{2}{c}{ Ragging } & Forest \\
\hline Accuracy & $\mathbf{0 . 9 9 8 4 1 3}$ & 0.983333 & 0.998016 & 0.997619 & $\mathbf{0 . 9 9 8 4 1 3}$ & $\mathbf{0 . 9 9 8 4 1 3}$ \\
Cohen Kappa & $\mathbf{0 . 9 9 7 0 2 5}$ & 0.968513 & 0.996281 & 0.995539 & $\mathbf{0 . 9 9 7 0 2 5}$ & $\mathbf{0 . 9 9 7 0 2 5}$ \\
Precision macro avg & $\mathbf{0 . 9 7 0 2 5 6}$ & 0.533845 & 0.901923 & 0.949516 & 0.943590 & 0.943590 \\
Precision weighted avg & 0.998714 & 0.971307 & 0.998291 & 0.998032 & $\mathbf{0 . 9 9 8 8 2 0}$ & $\mathbf{0 . 9 9 8 8 2 0}$ \\
Recall macro avg & 0.925000 & 0.502778 & 0.900000 & 0.917863 & $\mathbf{0 . 9 5 0 0 0 0}$ & $\mathbf{0 . 9 5 0 0 0 0}$ \\
Recall weighted avg & $\mathbf{0 . 9 9 8 4 1 3}$ & 0.983333 & 0.998016 & 0.997619 & $\mathbf{0 . 9 9 8 4 1 3}$ & $\mathbf{0 . 9 9 8 4 1 3}$ \\
f1 macro avg & 0.935889 & 0.488236 & 0.897671 & 0.921199 & $\mathbf{0 . 9 3 8 3 8 5}$ & $\mathbf{0 . 9 3 8 3 8 5}$ \\
f1 weighted avg & 0.998346 & 0.976372 & 0.998008 & 0.997599 & $\mathbf{0 . 9 9 8 4 1 7}$ & $\mathbf{0 . 9 9 8 4 1 7}$ \\
\hline
\end{tabular}




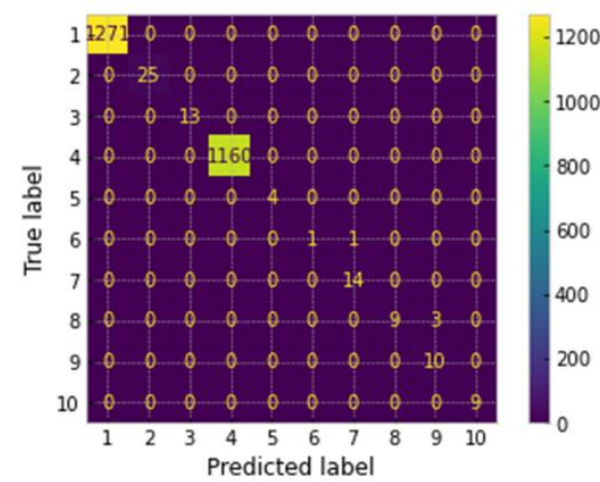

(a)

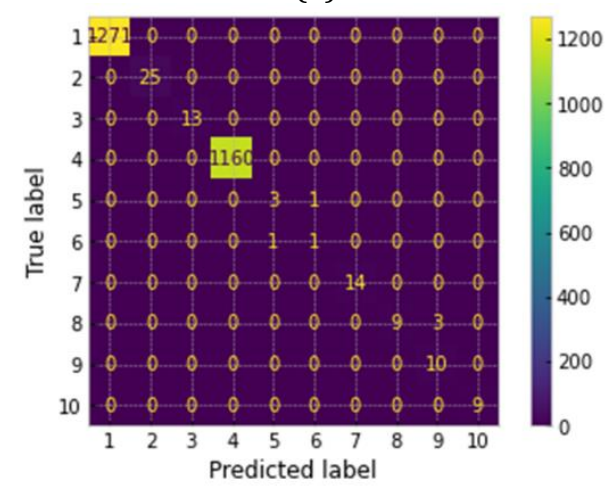

(c)

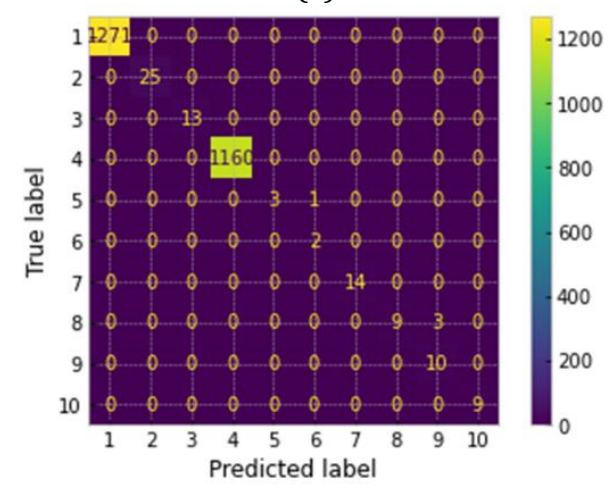

(e)

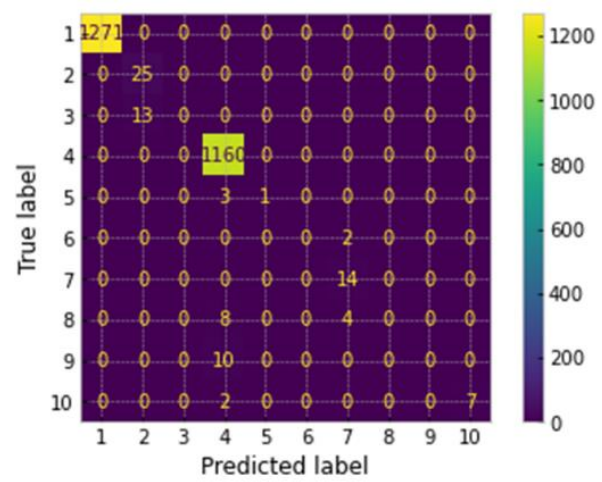

(b)

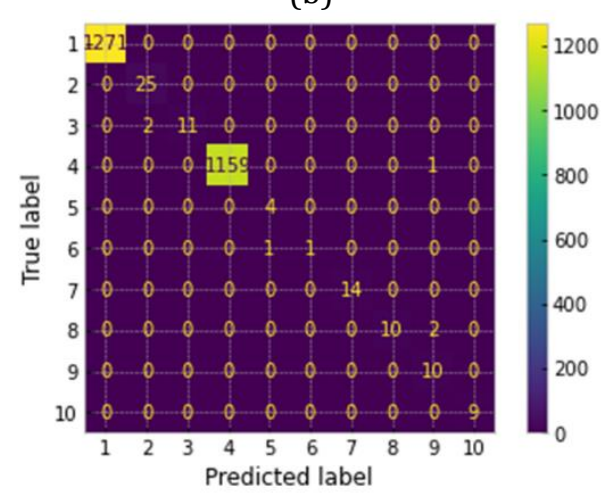

(d)

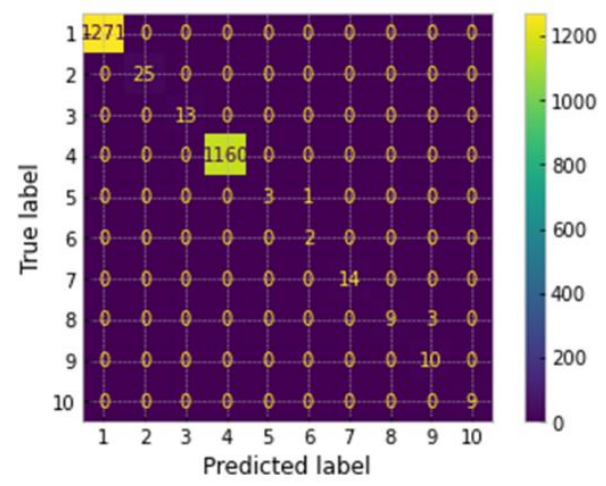

(f)

Figure 14. Confusion Matrix For Multi-Class Tests: (a) Logistic Regression; (b) Ridge Classifier; (c) K-NN ; (d) Bagging; (e) Random Forest; (f) SVM;

\section{Conclusion}

A critical step in PdM applications is continuous monitoring of equipment in operating conditions and detect/diagnose the fault events at an early stage. In this study, a widely used industrial equipment consisting of induction motors are analyzed and their common operational fault types in the industry are examined utilizing multiple data-driven ML models. Our results suggest that, by utilizing statistical time-domain features of the vibration signals, condition-based monitoring of fan motors can be efficiently implemented at a high success rate. Although only vibration data is utilized in this study, it is possible in the future to extend the study to multiple sensors and also deploy these ML models into an edge-device for continuous monitoring of equipment at the edge level.

\section{Acknowledgements}

This research is supported in part by 2232 International Fellowship for Outstanding Researchers Program of TÜBİTAK (Project No: 118C252) and also TÜBİTAK 1511 IOTOPRO Project (Project No: 1170452)

\section{Contribution of Researchers}

Mahmut KASAP contributed to the publication with the coding and writing of the article. Eyüp ÇİNAR contributed with project management, evaluation of methods, and review of the article. Ahmet YAZICI 
contributed to project consultancy, evaluation of methods and discussion of results and review of the article. Kemal ÖZKAN contributed to project consultancy, evaluation of methods, discussion of results and review of the article.

\section{Conflict of Interest}

No conflict of interest was declared by the authors.

\section{References}

Alpaydin, E. (2020). Introduction to machine learning: MIT press.

Benbouzid, M. E. H. (2000). A review of induction motors signature analysis as a medium for faults detection. IEEE Transactions on Industrial Electronics, 47(5), 984-993. doi:https://www.doi.org/10.1109/41.873206

Carvalho, T. P., Soares, F. A. A. M. N., Vita, R., Francisco, R. d. P., Basto, J. P., \& Alcalá, S. G. S. (2019). A systematic literature review of machine learning methods applied to predictive maintenance. Computers \& Industrial Engineering, 137, 106024. doi:https://doi.org/10.1016/j.cie.2019.106024

Deng, W., Yao, R., Zhao, H., Yang, X., \& Li, G. (2019). A novel intelligent diagnosis method using optimal LSSVM with improved PSO algorithm. Soft Computing, 23(7), 2445-2462. doi : https://doi.org/10.1007/ s00500-017-2940-9

Fengqi, W., \& Meng, G. (2006). Compound rub malfunctions feature extraction based on fullspectrum cascade analysis and SVM. Mechanical Systems and Signal Processing, 20(8), 2007-2021. doi:https://doi.org/10.1016/j.ymssp.2005.10.004

Glowacz, A. (2019). Fault diagnosis of single-phase induction motor based on acoustic signals. Mechanical Systems and Signal Processing, 117, 6580. doi : https://doi.org/10.1016/j.ymssp. 2018.07.044

Han, T., Jiang, D., Zhao, Q., Wang, L., \& Yin, K. (2017). Comparison of random forest, artificial neural networks and support vector machine for intelligent diagnosis of rotating machinery. Transactions of the Institute of Measurement and Control, 40(8), 26812693. doi : https://www.doi.org/10.1177 $\lcm{0142331217708242}$

Jimenez, J. J. M., Schwartz, S., Vingerhoeds, R., Grabot, B., \& Salaün, M. (2020). Towards multi-model approaches to predictive maintenance: A systematic literature survey on diagnostics and prognostics. Journal of Manufacturing Systems, 56, 539-557. doi:https://doi.org/10.1016/j.jmsy.2020.07.008

Katalin, Á. (2015). Fault Detection of the Electrical Motors Based on Vibration Analysis. Procedia Technology, 19, 547-553. doi: https://doi.org/10.1016/j.protcy.2015.02.078
Kumar, P., \& Hati, A. S. (2021). Review on Machine Learning Algorithm Based Fault Detection in Induction Motors. Archives of Computational Methods in Engineering, 28(3), 1929-1940. doi:https://www.doi.org/10.1007/s11831-020-09446-w

Liu, Z., Guo, W., Hu, J., \& Ma, W. (2017). A hybrid intelligent multi-fault detection method for rotating machinery based on RSGWPT, KPCA and Twin SVM. ISA Transactions, 66, 249-261. doi:https://doi.org/10.1016/j.isatra.2016.11.001

Moosavian, A., Ahmadi, H., Sakhaei, B., \& Labbafi, R. (2014). Support vector machine and K-nearest neighbour for unbalanced fault detection. Journal of Quality in Maintenance Engineering, 20(1), 65-75. doi:https://www.doi.org/10.1108/JQME-04-2012-0016

Rodriguez, P. J., Belahcen, A., \& Arkkio, A. (2006). Signatures of electrical faults in the force distribution and vibration pattern of induction motors. IEE Proceedings - Electric Power Applications, 153(4), 523-529. doi : https://www.doi.org/10.1049/ipepa:20050253

Saravanan, N., \& Ramachandran, K. I. (2009). Fault diagnosis of spur bevel gear box using discrete wavelet features and Decision Tree classification. Expert Systems with Applications, 36(5), 9564-9573. doi:https://doi.org/10.1016/j.eswa.2008.07.089

Saunders, C., Gammerman, A., \& Vovk, V. (1998). Ridge Regression Learning Algorithm in Dual Variables. Paper presented at the ICML.

Sullivan, G., Pugh, R., Melendez, A., \& Hunt, W. (2010). Operations \& Maintenance Best Practices - A Guide to Achieving Operational Efficiency (Release 3). Retrieved from https://www.osti.gov/biblio/1034595/

Sun, W., Chen, J., \& Li, J. (2007). Decision tree and PCAbased fault diagnosis of rotating machinery. Mechanical Systems and Signal Processing, 21(3), 1300-1317. doi:https://doi.org/10.1016/j.ymssp. 2006.06.010

Wang, D. (2016). K-nearest neighbors based methods for identification of different gear crack levels under different motor speeds and loads: Revisited. Mechanical Systems and Signal Processing, 70-71, 201-208. doi:https://doi.org/10.1016/j.ymssp. 2015.10.007

You, L., Fan, W., Li, Z., Liang, Y., Fang, M., \& Wang, J. (2019). A Fault Diagnosis Model for Rotating Machinery Using VWC and MSFLA-SVM Based on Vibration Signal Analysis. Shock and Vibration, 2019, 1908485. doi: https://www.doi.org/10.1155/2019/ 1908485

Zhou, Z., Wen, C., \& Yang, C. (2016). Fault Isolation Based on $k$-Nearest Neighbor Rule for Industrial Processes. IEEE Transactions on Industrial Electronics, 63(4), 2578-2586. doi:https://www.doi.org/10.1109/ TIE.2016.2520898 